\title{
La Ciudad Mestiza: Metrópolis Latinoamericanas Atrapadas Entre La Globalización Y La Inclusión Social
}

Pablo Ciccolella ${ }^{1}$

\section{Resumen}

Las transformaciones metropolitanas de las últimas décadas en América latina, han sido considerables. Ello genera la necesidad de una revitalizada discusión sobre la nueva naturaleza de la ciudad latinoamericana, intentando identificar rasgos comunes y singularidades en las grandes metrópolis de la región. Particularmente, este trabajo intenta discutir acerca de las nuevas tensiones que en esos nuevos escenarios metropolitanos están evidenciándose, tales como las dificultades del estado local para entender y metabolizar dichos cambios y desarrollar formas eficaces de intervención sobre el espacio urbano; el avance de un proyecto urbano hegemónico corporizado principalmente por el capital financiero e inmobiliario; y el agravamiento de una estructura socioeconómica-territorial históricamente desigual. Como trasfondo de estas tensiones, aparecen interrogantes significativos: ¿Es posible pensar en una ciudad latinoamericana competitiva, productiva y a su vez socialmente justa y territorialmente integradora? ¿Qué imágenes, qué modelos de ciudad van apareciendo como consecuencia de los acelerados cambios sufridos por las metrópolis, y, paralelamente, qué imaginarios, qué utopías urbanas están surgiendo? De un lado, se ubican los problemas de la comprensión de la naturaleza de los cambios en las formas, en la estructura, en las dinámicas, en las tendencias urbanas y de otro lado, los problemas referentes a las formas más adecuadas para intervenir y gestionar los sistemas socio-territoriales metropolitanos heredados de sucesivos procesos recientes de reestructuración.

Palabras clave: metrópolis, America latina, globalización, gobernabilidad, inclusión social

TAMOIOS. ANO VI. № 2, 2010 - ISSN 1980-4490 


\section{Neoliberalismo y territorios inestables}

El avance de la globalización neoliberal desde los años setenta, ha dado lugar a un creciente protagonismo del dinamismo económico y su preeminencia sobre otras dimensiones, tales como la política, la social o la cultural, si bien estas dimensiones y su evolución han acompañado y legitimado a las nuevas formas de relación entre economía y territorio.

Ello ha permitido un creciente protagonismo de la economía, y particularmente del capital transnacional que ha tendido a generar nuevas realidades territoriales de escala. $Y$ unas realidades territoriales sumamente lábiles, mutantes, a las que podríamos denominar escenarios o territorios inestables. La velocidad de los cambios, la aceleración de las transformaciones, está en la base explicativa de dicha inestabilidad de los territorios, se trate de territorios políticamente construidos (Estados-Nación, por ejemplo) o económicamente construidos (mercados comunes, uniones aduaneras, regiones económicas, etc.).

Como ya advertía hace tiempo Milton Santos, la aceleración contemporánea genera la sensación de que el presente huye (SANTOS, 1993, p.16 ) y con él las referencias que conocemos, las certezas que las estructuran; entre ellas, la de un territorio conocido y estable. Es decir, que la nueva condición geográfica que emerge es la de unos territorios inestables, o de unos escenarios geográficos que cambian rápidamente.

Evidentemente, este carácter inestable del territorio, plantea una dificultad cada vez mayor para entenderlo, para interpretar sus dinámicas, para construirlos intelectualmente y actuar sobre ellos a través de la planificación y el ordenamiento territorial. Se plantea una dificultad creciente para trazar los rasgos esenciales de los nuevos escenarios, que se tornan evanescentes. Caen entonces, buena parte de las categorías conceptuales, las herramientas metodológicas, los datos pierden el valor inercial -al que estábamos acostumbrados- y las posibilidades de experimentar la prospección, o cualquier forma de proyección a largo plazo se hace casi imposible -sino temeraria-, poniendo en crisis a todas las formas de planificación.

Otro rasgo singular de las nuevas tramas territoriales es la existencia de espacios que evolucionan a distintas velocidades. La selectividad territorial que caracteriza a esta etapa del capitalismo, provoca como resultado segmentos del territorio que se modernizan, evolucionan según los patrones económicoterritoriales predominantes, adoptan la forma, la estructura, el paisaje, las características urbanísticas y aún arquitectónicas propias de una época. Otros segmentos territoriales muestran una evolución más lenta hacia esos patrones de desarrollo. Por último, pueden observarse también espacios que no han sido prácticamente tocados por el llamado proceso de globalización. Y esto no sucede 
únicamente en territorios rurales, aislados o poco accesibles. Sucede en importantes fragmentos de las grandes ciudades, y en especial en los espacios que más compromiso tuvieron con el modelo económico y productivo anterior, en este caso, el fordista-desarrollista.

Desde la década pasada, las grandes metrópolis latinoamericanas enfrentan procesos de inversión y modernización sumamente selectivos en términos territoriales, que agudizan las desigualdades históricas y las formas de polarización social y segregación socioterritorial. Viejas y nuevas demandas insatisfechas plantean problemas de gobernabilidad que no siempre pueden ser afrontados con los procedimientos e instrumentos vigentes.

Los cambios experimentados, han remitido nuevamente a la discusión sobre los enfoques e instrumentos de la planificación reglamentaria y prospectiva y han difundido, entre otras, las metodologías de la planificación estratégica y participativa. Más allá de las variadas experiencias de planificación urbana, más allá de sus éxitos y fracasos, podríamos afirmar que uno de los actuales desafíos de la planificación urbana es intervenir territorios cada vez más inestables y complejos, sujetos a dinámicas multiescalares y multiterritoriales, con nuevos procesos de fragmentación y segregación socioterritorial.

En definitiva, lo que tenemos ante nuestra mirada es una realidad territorial marcada por una creciente inestabilidad. Inestabilidad causada por aquello que Milton Santos llamaba aceleración contemporánea (SANTOS, 1993, p. 15-16), es decir, aceleración de los procesos económicos, de los procesos territoriales, lo que lleva a la generación de nuevos escenarios de creciente inestabilidad.

Así la fluidez y su expresión espacial: los flujos; avanzan sobre la geografía de las estructuras fijas y contiguas. Se va insinuando cada vez con mayor claridad que se evoluciona hacia una geografía de los flujos. Sin embargo, los lugares, a su vez, ganan en importancia, en espesor, en especificidad, en un paradójico enriquecimiento de las condiciones locales. Es lo que Santos denominó "el retorno del territorio" (SANTOS, 1994).

Los factores "extraeconómicos" (social, histórico, político, cultural, territorial, institucional, y particularmente la dimensión territorial, van adquiriendo mayor importancia en la nueva relación entre economía y territorio (VELTZ, 1996, p. 11). Aquí se observa otra paradoja. Si bien el dinamismo económico es el dominante en esta etapa del capitalismo, frente al dinamismo político y social de la etapa anterior; ese dinamismo económico dominante, para serlo, se apoya en los factores extraeconómicos mencionados. Se nutre especialmente de las "condiciones del territorio concreto", de sus recursos humanos, de su capacidad innovadora, de sus instituciones, de sus tradiciones y trayectorias productivas, etc.) 
Así el mundo no aparece como una simple red de lugares competitivos como células de la economía global, ni como una red de megaciudades o ciudades globales, ni tampoco como un mosaico de zonas. Es algo mucho más complejo. Es, básicamente un tejido de redes y lugares, de flujos y de estructuras fijas, de estructuras geoeconómicas con tiempos a velocidades diferentes, mezcla de orden y caos, de racionalidad ordenadora y de intensas transformaciones permanentes, de escenarios territoriales inestables.

Las nuevas formas de producción y articulación del espacio constituyen a la vez un rasgo distintivo y uno de los factores explicativos de las nuevas formas de metropolización. Se ha pasado de un espacio metropolitano compacto, que avanzaba en forma de "mancha de aceite", con una morfología y bordes bastante bien definidos, hacia un crecimiento metropolitano en red, conformando una verdadera ciudad-región, de bordes difusos, policéntrica, constituyendo en algunos casos, verdaderas megalópolis o archipiélagos urbanos. En otras palabras, se pasa de territorios estructurados fundamentalmente en base a la articulación horizontal y contigua de los lugares o regiones, a un territorio estructurado tridimensionalmente y verticalmente por medio de redes y en forma de red (CICCOLELLA, 1999, p. 8).

Las relaciones de contigüidad en el nuevo contexto significan poco en los nuevos procesos de producción y articulación del espacio. La forma en que se articula el espacio en el capitalismo flexible no es predominantemente horizontal. Las "verticalidades" como plantea Santos, juegan un rol muy fuerte como articuladoras en un espacio que tiende a estructurarse a partir de relaciones verticales y piramidales que se superponen a las relaciones horizontales, de contigüidad y habitualmente las hegemonizan (SANTOS, 1996, p. 222). Si bien las condiciones "locales" pueden potenciar un escenario territorial más atractivo que otro, nada impide que una ciudad (o una empresa) pueda prosperar en medio de una región que declina, en tanto sea capaz de inscribirse en una malla de relaciones funcionales, organizacionales e informacionales multiescalares, como resultado del pasaje de un territorio de zonas o lugares a un territorio de redes o flujos (VELTZ, 1994b, p. 29).

\section{La ciudad como condominio privado para ricos}

Buenos Aires muestra el drama propio de varios lugares de América latina, que se han convertido en locus privilegiados del mercado global de bienes raíces, con su consiguiente burbuja especulativa y su correspondiente inflación de valores del suelo.

El precio promedio del suelo urbano pasó de 558 US $\$ / \mathrm{m}^{2}$ en diciembre de 2001 a 1.014 US $\$ / \mathrm{m}^{2}$ en diciembre de 2006 , luego de superar una abrupta caída en el mes de junio de 2002 donde los valores promedio bajaron a 214 US $\$ / \mathrm{m}^{2}$. Esta evolución de los precios inmobiliarios muestra no solo una rápida 
recuperación sino la duplicación, en algunos barrios, de los valores record alcanzados en la década de los noventa (BAER, 2006).

La misma evolución siguieron las unidades residenciales a estrenar en edificios multivivienda de alto estándar. Con valores promedio de $1.200 \mathrm{US} \$ / \mathrm{m}^{2}$ en 1995 se pasa a 2.000 US $\$ / \mathrm{m}^{2}$ en el 2005 , llegando en la actualidad a valores superiores a los 4.000 US $\$ / \mathrm{m}^{2}$ en edificios y localizaciones privilegiadas

La fractura socioterritorial también se expresa en los precios inmobiliarios: el valor del $\mathrm{m}^{2}$ de vivienda a estrenar en los barrios del norte de la ciudad, es diez veces mas alto que en los barrios del sur. En 2001 se necesitaban 43 salarios promedio para comprar un departamento de un dormitorio en el norte y 28 en el sur. Esta relación para junio de 2006 aumentó a 182 y 87 salarios medios, respectivamente (BAER, 2006).

Los procesos de densificación y verticalización de la ciudad de Buenos Aires asumen así una doble dinámica. Por un lado hay una densificación, modernización y ampliación del espacio de oficinas en el área central de la ciudad de Buenos Aires (CICCOLELLA y LUCIONI, 2003) y por otro la densificación y verticalización del espacio residencial dirigido a los sectores de más altos ingresos en los distritos urbanísticos ya mencionados (MIGNAQUI Y SZAJNBERG, 2003).

Buenos Aires es una ciudad bastante singular, en el marco de las grandes metrópolis latinoamericanas. Allí, la vanguardia en materia de movimientos y organizaciones sociales no está solamente, ni principalmente representada por sectores populares, sino por ciertos sectores de clase media que vienen comportándose como las más eficaces luchadoras contra la especulación inmobiliaria en algunos barrios de la ciudad, aunque con una argumentación y un discurso que podría ser catalogado como burgués: mantener la identidad arquitectónica y urbanística del barrio.

El proyecto hegemónico ha encontrado a través de la densificación y verticalización de los distritos urbanísticos residenciales y comerciales no sólo una herramienta para la realización del capital en un espacio-tiempo limitado sino también para reconfigurar la ciudad haciéndola cada vez más inaccesible para la mayoría de la población. La elitización de algunos fragmentos de la ciudad, los proyectos en carpeta para las pocas reservas de tierras vacantes e inmuebles públicos que esperan una definición respecto de sus usos futuros y asignación social, reafirman la debilidad institucional y fragilidad de las políticas territoriales para orientar el desarrollo futuro de la ciudad.

Miles de personas locales y extranjeras se enriquecieron increíblemente comprando suelo urbano barato en 2002 y ahora multiplicando por 5 su capital, sin contar los beneficios de las operaciones de desarrollo inmobiliario de alto estándar. 
Con semejanzas y diferencias, en todas partes el modelo neoliberal ha generado: mayor libertad de acción al capital como ordenador territorial preponderante; una creciente influencia del capital oligopólico sobre las transformaciones del territorio; y un comportamiento de las ciudades como si fuesen firmas compitiendo entre si para atraer inversiones a cualquier costo.

Esto, a su vez ha generado un fuerte impacto sobre territorios metropolitanos que van de reestructuración en reestructuración y quedan atrapados entre tensiones aparentemente contradictorias entre desconcentración y recentralización, entre globalización-neoliberalización e inclusión social

Actualmente, las grandes metrópolis parecen tener tres actores preponderantes: el primero -en una posición dominante-, el capital global, con sus múltiples conexiones nacionales y locales; en segundo lugar, el Estado, en una situación que podría definirse como una mezcla de ineficacia y complicidad con las dinámicas hegemónicas; $y$, por último, los sectores populares, que en los últimos años parecen ir ganando terreno, al menos simbólicamente, en materia de organización y capacidad de lucha. La inclusión de estos sectores sociales es sin duda uno de los desafíos pendientes y futuros en la agenda del desarrollo metropolitano

Ya a esta altura de los acontecimientos es bueno preguntarnos si resulta imprescindible para el bien común, para el bienestar general, que nuestras ciudades entren en los patrones de la ciudad global. Para qué? En qué han mejorado México o San Pablo, desde que son consideradas por los sectores hegemónicos ciudades globales de segundo orden?

Estas evidencias mueven a replantear los supuestos, las estrategias, las políticas y las herramientas o acciones del desarrollo urbano en el marco de una etapa del capitalismo dominada por tendencias a un mayor incremento de la fractura social y territorial, a un divorcio entre el espacio de las empresas y el de la gente, entre las élites y las mayorías, entre los segmentos modernizados, competitivos y globalizados de las ciudades y los nuevos arrabales.

Se trata de repensar la ciudad como un ámbito de socialización, de convivencia de sectores sociales, de distintos niveles de formación profesional y cultural, como un ámbito de producción y de trabajo, donde la actividad industrial y la extensa gama de servicios a la producción y al consumo pueden constituir una nueva base económica para la ciudad, a partir de la incorporación intensiva de conocimiento, creatividad, tecnología e inteligencia no sólo a la producción sino a la propia gestión del espacio urbano.

Sin embargo, lamentablemente, los hechos parecen ir en un sentido contrario. El sistema capitalista actual se ha transformado en un tipo de capitalismo que podríamos denominar capitalismo metropolitano, en tanto que el 
capital se realiza a través del territorio urbano-metropolitano y en la medida en que el capital privado aparece como ordenador territorial preponderante basándose en los múltiples recursos o ventajas competitivas de cada porción del territorio, extrayendo las plusvalías más cuantiosas precisamente de las singularidades, y especificidades que hacen de cada territorio el lugar óptimo para cada tipo de producción. Por otra parte, como sostiene David Harvey, el capitalismo actual compra influencia política y es constitutivamente corrupto y corruptor en términos políticos (HARVEY, 2007, p. 24). Precisamente, el factor central de los cambios económicos, sociales, políticos, culturales y territoriales parece ser una convergencia entre los avances científico-tecnológicos, una nueva fase de explotación extrema de la fuerza de trabajo y la capacidad del capital para comprar influencia política. En algún sentido, este capitalismo metropolitano, se parece a las formas más primitivas del capitalismo del siglo XVIII.

La transformación de grandes ciudades en centros financieros globales como Nueva York, Londres, Tokio, Frankfurt, y, en un futuro próximo, seguramente también San Pablo, Santiago, México, Buenos Aires, Shangai, Mumbai, El Cairo, etc. va haciendo progresivamente imposible a los sectores populares y ciertas clases medias vivir en los núcleos centrales de las grandes conurbaciones. El dinero especulativo financiero-inmobiliario esta "comprando" los mejores lugares de las ciudades y convirtiéndolas en gigantescos condominios cerrados y desplazando hacia las periferias más hostiles no sólo a los pobres sino también a los sectores medios. Esto es ya una realidad tangible en Nueva York y Londres y una tendencia cierta en las principales ciudades del mundo y de América latina, como se ha visto en el caso de Buenos Aires.

Esto representa un gran problema y desafío intelectual, político y metodológico. Ya no es sólo la clase trabajadora, en términos clásicos, la que está siendo desposeída y desplazada. Las izquierdas políticas y académicas deberían prestar más atención a estos nuevos fenómenos, relativizando los conceptos clásicos e intentando acumular energía para unificar los diferentes movimientos sociales y las formas de resistencia y lucha.

\section{Revisitando la metrópolis latinoamericana: la ciudad mestiza}

Las metrópolis se han ido transformando en espacios de gran magnitud y complejidad, que el poder del capital de gran escala, desmonta en fragmentos de ciudad o de barrios. La lógica del capital fragmenta una vez más el territorio, pero esta vez de un modo hiperselectivo, agravando la ya histórica relación contradictoria con el espacio, destruyendo viejos órdenes y construyendo otros cada vez más aceleradamente priorizando demandas completamente apartadas de los intereses populares.

Al lado de la ciudad exitosa, continúa estando la otra ciudad, la ciudad popular, la que busca salidas que garanticen la supervivencia. Una ciudad que 
esta viva y necesita soluciones. Lo realmente importante es que nuestras ciudades sean buenos lugares para vivir. Ello no pareciera depender obsesivamente, necesariamente, del capital global y las inversiones extranjeras directas. Más bien dependen de la historia de nuestras ciudades, del tipo de gestión que en ellas se realice, del lugar que el ciudadano común ocupe en las políticas públicas. Vamos a continuar restando importancia al hecho de que al lado de los barrios exitosos de la ciudad ha crecido también la ciudad popular, alternativa, la economía informal, incluso a veces competitiva?

La ciudad global en América latina sólo está representada por ciertos fragmentos privilegiados de la ciudad que el discurso político, el de los media, y aún a veces el propio discurso académico mistifica por el conjunto de la ciudad

Nuestras ciudades se parecen en sus problemas, lo cual es una fortaleza para una posible lucha común en el futuro, pero también, desgraciadamente, se parecen en sus fragmentos más globalizados: los barrios privados, los distritos de comando, los grandes centros comerciales y de ocio, etc. Nuestras ciudades se empobrecen con ese tipo de similitudes. La potencialidad y fortaleza de nuestras ciudades está precisamente en los problemas que nos unen, en nuestra historia común, en el marco de nuestra diversidad cultural y de nuestros movimientos sociales.

Los territorios urbanos actuales representan un lugar y un momento donde esos actores no hegemónicos pueden emerger, lo que va a obligar a repensar y revisitar el contenido y las herramientas futuras de la gestión urbana, incluyéndolos e integrándolos. Parafraseando a García Canclini, podemos imaginar a las ciudades latinoamericanas saliendo y entrando convenientemente de la globalización (GARCIA CANCLINI, 1992). Tomando de ella lo que puede servir, pero sin involucrarse dependientemente en la misma.

En las metrópolis latinoamericanas han cambiado, la base económica, los mercados de trabajo, el paisaje urbano, los agentes decisivos de estructuración del espacio urbano, los rasgos morfológicos, las formas de expansión y crecimiento, las relaciones sociales, las formas de gestión urbana, los hábitos culturales, etc. Sin embargo, las singularidades, la resistencia inercial de las identidades urbanas, las especificidades de estos cambios, en tanto procesos territoriales que engloban, por ejemplo, la recuperación del patrimonio y de la memoria territorial, conducen a un escenario híbrido. Quizás deberemos acostumbrarnos a estudiar a las ciudades como resultado de procesos sobreimpuestos violentamente sobre territorios heredados: ciudades híbridas o mestizas.

Evidentemente estamos frente a un proceso de mestizaje territorial que está dando lugar a una nueva naturaleza de la ciudad latinoamericana que denominamos ciudad mestiza, en razón de una serie de atributos evidentes que podemos esquematizar en base a los siguientes procesos: 
- Yuxtaposición de rasgos preibéricos, coloniales, agroexportadores, industrialistas y neoliberal-posmodernos

- Exacerbación de las contradicciones y contrastes

- Agravamiento de las desigualdades sociales, económicas y territoriales, históricamente consolidadas

- Incremento de la fragmentación por una selectividad territorial creciente del capital y las inversiones

- Un mayor proceso de hibridación cultural

Pero en un plano menos evidente, las transformaciones aludidas, que nos llevan a considerar a las grandes ciudades latinoamericanas como ciudades mestizas, más que nada devienen de la resolución traumática y parcial de la antítesis desarrollismo/neoliberalismo, o lo que sería lo mismo: la no resolución de las tendencias hacia metrópolis sustitutivas o hacia metrópolis en proceso de globalización. Analizando los diferentes casos metropolitanos podría decirse que, en algunos, los cambios recientes son simplemente una aceleración de tendencias presentes en la etapa desarrollista. En este sentido, los cambios podrían ser profundos pero no estructurantes de una realidad metropolitana completamente nueva, haciendo que la ciudad desarrollista evolucione y se reproduzca, pero sin transformarse radicalmente en una ciudad diferente (CICCOLELLA, 2007: P. 134139). Quizá Montevideo, Lima, Buenos Aires, Rio o Bogotá representarían bastante bien esta hipótesis. Otros casos sugieren transformaciones territoriales metropolitanas suficientemente profundas y estructurales como para pensar en una ruptura radical con las características de la ciudad desarrollista latinoamericana. Es decir, un proceso de cambio en el cual la globalización arrasa formas y estructuras espaciales preexistentes y moldea una nueva metrópolis: la ciudad del capitalismo global. Santiago de Chile, Sao Paulo y México, bien podrían ilustrar esta hipótesis.

\section{Reflexiones finales}

Valdría la pena estudiar la posibilidad de la construcción de una ciudad más equitativa, justa y solidaria. Buena parte de la sociedad así lo está demandando de diversas formas. Ya sea la formación creciente de economías alternativas y creativas, o la formación y resistencia de nuevos movimientos sociales urbanos, parecen demandar otro orden, y más allá de cualquier posicionamiento ideológico, se debería prestar atención a estos fenómenos. 
Se trata básicamente de centrar el desarrollo urbano en la regeneración del tejido socioproductivo bajo condiciones de modernización, capacitación de recursos humanos, incorporación de tecnología, más que el desarrollo de servicios banales y las operaciones cosméticas de marketing urbano. El objetivo central de las acciones deberían ser la mejora de la calidad de vida de los ciudadanos y de sus capacidades, priorizando el fortalecimiento de las redes solidarias, tanto a nivel de los ciudadanos como de las empresas y las instituciones, mejorando paralelamente a las propias instituciones. En síntesis, más redes solidarias y menos parques temáticos, shopping centers y centros de ocio, es decir menos artefactos de la globalización y de la banalidad y más aplicación de creatividad e inteligencia transformadoras

Resulta necesario entonces construir una nueva teoría crítica de la ciudad, desde el sur, desde América latina, a partir de la abundante evidencia empírica acumulada en los últimos años, generando nuevas o renovadas categorías conceptuales y recursos metodológicos. A veces el problema no es la denominación de fenómenos y procesos urbanos (tarea en la que hemos perdido demasiado tiempo), sino la manera en como los interpretamos y los valoramos desde nuestra realidad latinoamericana

Es preciso que nos formulemos nuevas preguntas, como por ejemplo: Qué hacer con estos cambios? Resulta posible superar el supuesto antagonismo entre performance económica e inclusión y progreso social? Cómo mejorar la comprensión de la naturaleza de estos cambios y de la propia naturaleza de la ciudad actual y cómo rediseñar políticas de intervención sobre unos sistemas socioterritoriales metropolitanos tan complejos y cambiantes

Los cambios económicos, sociales y territoriales de las últimas décadas no deben ser naturalizados. Debemos pasar a un segundo plano o al menos matizar el discurso y los relatos de la globalización en nuestras ciudades (tareas en la que también hemos perdido demasiado tiempo), profundizando en las nuevas formas de fractura socioterritorial, desmitificando la narrativa política, mediática y científica falaz que extiende al conjunto de nuestras metrópolis, la experiencia supuestamente exitosa de algunos fragmentos de nuestras ciudades.

Debemos revisar las alternativas "fashion" de neoplanificación urbanametropolitana como la planificación estratégica acríticamente adoptada "llave en mano" en toda América latina. Ello debe contribuir al actual proceso de emergencia y reescalamiento de nuevos poderes opuestos al orden neoliberal: el Estado Nacional y los nuevos movimientos sociales urbanos, en varios de nuestros países.

En fin deberemos rediscutir el rol del Estado y de sus diversas escalas en materia de intervención urbana-metropolitana para propender a unas ciudades territorialmente equilibradas y socialmente integradoras, con más redes solidarias 
y productivas y menos artefactos de la globalización. Resulta decisiva la recuperación de la esfera nacional en los asuntos metropolitanos. Los municipios no tienen entidad suficiente para contener el avance de los proyectos hegemónicos de ciudad, y habitualmente hay gran dificultad para lograr que aquellos se articulen entre sí.

Debe ser estudiadas y diseñadas alternativas superadoras de la engañosa oposición entre una ciudad productiva que gane en capacidad competitiva y a la vez sea integradora y solidaria. En tanto no haya alternativa al capitalismo, no se puede condenar a la ciudad a permanecer en una economía de supervivencia. Ninguna teoría política puede avalar que la respuesta al capitalismo sea sacar a nuestras ciudades del contexto y la dinámica económica y tecnológica mundial. A la globalización no se la derrota ignorándola, sino con sus propias armas...

Necesitamos también recuperar una mística de producción del conocimiento que aún con el riesgo de cierta ideologización nos permita imaginar instrumentos conceptuales y de intervención pública para colaborar en la construcción de una ciudad mejor para todos.

En fin, Identificar las problemáticas comunes y también las potencialidades que articulan el futuro de nuestras ciudades en el marco de la diversidad cultural latinoamericana.

Nuestras ciudades se parecen (se empobrecen, en términos culturales) en sus fragmentos globalizados y globalizantes. Pero se diferencian por su identidad, por sus rasgos idiosincráticos, su cultura y su historia, por sus practicas sociales, por sus movimientos sociales y por la mezcla de ideas que flota en sus bares, en sus plazas, en sus esquinas, en sus estadios de fútbol y, sobre todo por la bronca en las calles y la rebelión de las masas en los sitios icónicos de las luchas populares históricas. Seguramente sería exitoso aprovechar esa riqueza inmensa para potenciar las luchas sociales urbanas en una red de solidaridad de los sectores populares a través del continente.

En la medida en que el Estado nacional no retome el comando de los procesos socio-territoriales y oriente las tendencias de desarrollo urbano con un sesgo integrador y solidario, apuntalando tanto la fragilidad de los gobiernos locales como a las demandas que la sociedad civil expresa a través de los nuevos movimientos sociales, no resulta posible imaginar un futuro con justicia social y equidad para nuestras ciudades. Los vigorosos rumbos trazados por el capital hegemónico en la edad de oro del neoliberalismo en América latina, sólo podrán ser desandados a través de un proceso de convergencia entre las mayorías populares y un proyecto político que integre las escalas nacional y local.

Nuestra hipótesis admite la existencia de otros caminos y alternativas de desarrollo y progreso urbano en el mejor y más amplio sentido de estas 
expresiones. El desafío es construir discursos, conceptos e instrumentos analíticos y operativos alternativos, que eludan el canto de sirena del pensamiento único sobre la ciudad y la planificación urbana. En fin, identificar las problemáticas comunes y también las potencialidades que articulan el futuro de nuestras ciudades en el marco de la diversidad cultural latinoamericana.

\section{Notas:}

1 - Doctor en Geografía, Ordenamiento Territorial y Urbanismo. Director del Instituto de Geografía de la Facultad de Filosofía y Letras de la Universidad de Buenos Aires. Director del Programa de Desarrollo Territorial y Estudios Metropolitanos (PDTEM) Profesor e Investigador en la FFyL - UBA. pablociccolella@arnet.com.ar

Las grandes metrópolis latinoamericanas se han ido transformando en territorios de considerable magnitud y complejidad, que el poder económico tiende a desmontar en fragmentos de ciudad o de barrios, con una lógica cada vez más selectiva y acelerada, destruyendo viejos órdenes territoriales y construyendo otros, sobre cuyas claves aún no hemos reflexionado acabadamente, aunque ya poseemos un importante conocimiento empírico sobre estos procesos.

El propio discurso académico parece estar en un callejón sin salida. Las reflexiones se han estancado en torno al discurso y a los relatos de la globalización de nuestras ciudades. Es necesaria una reflexión desde el sur, que vaya más allá, profundizando sobre las nuevas formas de fractura socio-territorial, desmitificando la narrativa política, mediática y científica falaz que extiende al conjunto de nuestras metrópolis, la experiencia supuestamente exitosa de algunos fragmentos de nuestras ciudades.

\section{Bibliografia}

BAER, Luis. Valorización inmobiliaria y dificultades para acceder a la vivienda en la Ciudad de Buenos Aires luego de la crisis de 2001/2002. Buenos Aires: Instituto de Geografía-UBA, 2007

CICCOLELLA, Pablo. Globalización y dualización en la región metropolitana de Buenos Aires. Grandes inversiones y reestructuración socioterritorial en los años noventa. Revista EURE, Santiago, Vol. XXV - № 76, p. 5-27, diciembre 1999

CICCOLELLA, Pablo y LUCIONI, Nora. La ciudad corporativa. Nueva arquitectura empresarial, redefinición de la centralidad y surgimiento de una red de distritos de comando en la Región Metropolitana de Buenos Aires, in De Mattos, Carlos et. al. (Editores) Gobernanza, Competitividad y Redes: la gestión en las ciudades del siglo $X X I$. Santiago: Instituto de Estudios Urbanos y territoriales Pontificia Universidad Católica de Chile, Colección RIDEAL - EURE libros, 2005, p. 185-209 
CICCOLELLA, Pablo. Metrópolis latinoamericanas: territorios subregulados, espacios del capital? In Aguilar, Adrián, G. (Coordinador) Las grandes aglomeraciones y su periferia regional. Experiencias en Latinoamérica y España. México: CONACYT- HCD - IG / UNAM - M.A. Porrua Editor, 2006, p.305-324

CICCOLELLA, Pablo. Transformaciones recientes en las metrópolis latinoamericanas. In Fernandez Casó, V. y Gurevich, R. (coord.) Geografía, nuevos temas, nuevas preguntas, Buenos Aires: Ed. Biblos, 2007, p.125.145.

GARCIA CANCLINI, Néstor. Culturas Híbridas. Estrategias para entrar y salir de la modernidad. Buenos Aires: Ed. Sudamericana, 1992

HARVEY, David. Los espacios del capitalismo global", Revista Espacios, Buenos Aires: $N^{\circ} 35$, Facultad de Filosofía y Letras de la Universidad de Buenos Aires, 2007, p. 20-32

MIGNAQUI, lliana y SZAJNBERG, Daniela. Tendencias en la organización del espacio residencial en la región metropolitana de Buenos Aires en los noventa. In Bertoncello, Rodolfo y Alessandri Carlos, Ana Fani (Compiladores) Procesos territoriales en Argentina y Brasil. Buenos Aires: Instituto de Geografía, Facultad de Filosofía y Letras, Universidad de Buenos Aires, Buenos Aires, 2003, p. 91-115

POMA, Lucio. La nueva competencia territorial. In Boscherini, F. y Poma, L (compiladores) Territorio, conocimiento y competitividad. El rol de las instituciones en el espacio global. Buenos Aires: Miño y Dávila Editores, 2000, p. 39-76

SANTOS, Milton. A aceleracao contemporânea: tempo-mundo e espaco-mundo In Santos M. et al. (Org.) O Novo Mapa do Mundo. Fim de século e globalizacao. Sao Paulo: Hucitec-Anpur, 1993, p. 15-22

SANTOS, Milton. O retorno do território- In Santos, M. y otros (org.) Território. Globalizacao e Fragmentacao, Sao Paulo: Hucitec, 1994, p. 15-20

SANTOS, Milton. A natureza do espaço. Ténica e tempo. Razao e emoçao. Sao Paulo: HUCITEC, 1996

SASSEN, Saskia. Sociología de la globalización. Buenos Aires: Katz Editor, 2007

VELTZ, Pierre. Des territoires pour apprendre et innover. Paris: Éditions de l'aube, 1994

VELTZ, Pierre. Mondialisation, Villes et Territoires. L'Économie d'Archipel. Paris : Presses Universitaires de France, 1996 